\title{
Exploiting Semantic Information for HPSG Parse Selection
}

\author{
Sanae Fujita, ${ }^{\odot}$ Francis Bond, $^{\oplus}$ Stephan Oepen, ${ }^{*}$ Takaaki Tanaka ${ }^{\odot}$ \\ $\odot$ \{sanae, takaaki\}@cslab.kecl.ntt.co.jp, ' bond@ieee.org, \$ oe@ifi.uio.no \\ $\odot$ NTT Communication Science Laboratories, Nippon Telegraph and Telephone Corporation \\ - National Institute of Information and Communications Technology (Japan) \\ * University of Oslo, Department of Informatics (Norway)
}

\begin{abstract}
In this paper we present a framework for experimentation on parse selection using syntactic and semantic features. Results are given for syntactic features, dependency relations and the use of semantic classes.
\end{abstract}

\section{Introduction}

In this paper we investigate the use of semantic information in parse selection.

Recently, significant improvements have been made in combining symbolic and statistical approaches to various natural language processing tasks. In parsing, for example, symbolic grammars are combined with stochastic models (Oepen et al., 2004; Malouf and van Noord, 2004). Much of the gain in statistical parsing using lexicalized models comes from the use of a small set of function words (Klein and Manning, 2003). Features based on general relations provide little improvement, presumably because the data is too sparse: in the Penn treebank standardly used to train and test statistical parsers stocks and skyrocket never appear together. However, the superordinate concepts capital ( $\supset$ stocks) and move upward ( $\supset$ sky rocket) frequently appear together, which suggests that using word senses and their hypernyms as features may be useful

However, to date, there have been few combinations of sense information together with symbolic grammars and statistical models. We hypothesize that one of the reasons for the lack of success is that there has been no resource annotated with both syntactic and semantic information. In this paper, we use a treebank with both syntactic information (HPSG parses) and semantic information (sense tags from a lexicon) (Bond et al., 2007). We use this to train parse selection models using both syntactic and semantic features. A model trained using syntactic features combined with semantic information outperforms a model using purely syntactic information by a wide margin $(69.4 \%$ sentence parse accuracy vs. $63.8 \%$ on definition sentences).

\section{The Hinoki Corpus}

There are now some corpora being built with the syntactic and semantic information necessary to investigate the use of semantic information in parse selection. In English, the OntoNotes project (Hovy et al., 2006) is combining sense tags with the Penn treebank. We are using Japanese data from the Hinoki Corpus consisting of around 95,000 dictionary definition and example sentences (Bond et al., 2007) annotated with both syntactic parses and senses from the same dictionary.

\subsection{Syntactic Annotation}

Syntactic annotation in Hinoki is grammar based corpus annotation done by selecting the best parse (or parses) from the full analyses derived by a broadcoverage precision grammar. The grammar is an HPSG implementation (JACY: Siegel and Bender, 2002), which provides a high level of detail, marking not only dependency and constituent structure but also detailed semantic relations. As the grammar is based on a monostratal theory of grammar (HPSG: Pollard and Sag, 1994), annotation by manual disambiguation determines syntactic and semantic structure at the same time. Using a grammar 
helps treebank consistency - all sentences annotated are guaranteed to have well-formed parses. The flip side to this is that any sentences which the parser cannot parse remain unannotated, at least unless we were to fall back on full manual mark-up of their analyses. The actual annotation process uses the same tools as the Redwoods treebank of English (Oepen et al., 2004).

A (simplified) example of an entry is given in Figure 1. Each entry contains the word itself, its part of speech, and its lexical type(s) in the grammar. Each sense then contains definition and example sentences, links to other senses in the lexicon (such as hypernym), and links to other resources, such as the Goi-Taikei Japanese Lexicon (Ikehara et al., 1997) and WordNet (Fellbaum, 1998). Each content word of the definition and example sentences is annotated with sense tags from the same lexicon.

There were 4 parses for the definition sentence. The correct parse, shown as a phrase structure tree, is shown in Figure 2. The two sources of ambiguity are the conjunction and the relative clause. The parser also allows the conjunction to combine 電車 densha and $ᄉ$ hito. In Japanese, relative clauses can have gapped and non-gapped readings. In the gapped reading (selected here), 人 hito is the subject of 運転 unten “drive". In the non-gapped reading there is some underspecified relation between the modifee and the verb phrase. This is similar to the difference in the two readings of the day he knew in English: "the day that he knew about" (gapped) vs "the day on which he knew (something)" (nongapped). Such semantic ambiguity is resolved by selecting the correct derivation tree that includes the applied rules in building the tree (Fig 3).

The semantic representation is Minimal Recursion Semantics (Copestake et al., 2005). We simplify this into a dependency representation, further abstracting away from quantification, as shown in Figure 4. One of the advantages of the HPSG sign is that it contains all this information, making it possible to extract the particular view needed. In order to make linking to other resources, such as the sense annotation, easier predicates are labeled with pointers back to their position in the original surface string. For example, the predicate densha_n_1 links to the surface characters between positions 0 and 3:電車.

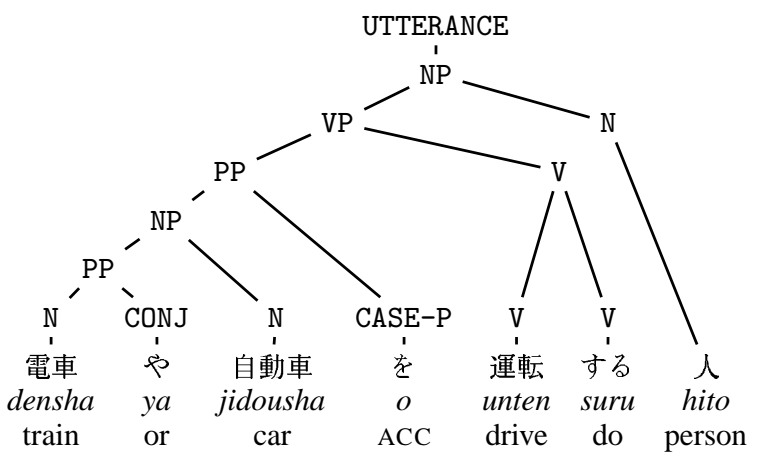

運転手 1 “chauffeur”: "a person who drives a train or car"

Figure 2: Syntactic View of the Definition of 運転 手 1 untenshu "chauffeur"

e2: unknown $<0: 13>$ [ARG $x 5$ :_hito_n]

$x 7:$ densha_n_ $1<0: 3>[]$

$\mathrm{x} 12:$ _jidousha_n $<4: 7>[]$

x13:_ya_p_conj $\langle 0: 4>$ [LIDX $x 7$ :_densha_n_1,

RIDX x12:_jidousha_n]

e23:_unten_s_2<8:10>[ARG1 x5:_hito_n]

e23:_unten_s_2<8:10>[ARG2 x13:_ya_p_conj]

Figure 4: Simplified Dependency View of the Definition of 運転手 1 untenshu "chauffeur"

\subsection{Semantic Annotation}

The lexical semantic annotation uses the sense inventory from Lexeed (Kasahara et al., 2004). All words in the fundamental vocabulary are tagged with their sense. For example, the word 大きい ookii "big" (of example sentence in Figure 1) is tagged as sense 5 in the example sentence, with the meaning "elder, older".

The word senses are further linked to semantic classes in a Japanese ontology. The ontology, GoiTaikei, consists of a hierarchy of 2,710 semantic classes, defined for over 264,312 nouns, with a maximum depth of 12 (Ikehara et al., 1997). We show the top 3 levels of the Goi-Taikei common noun ontology in Figure 5. The semantic classes are principally defined for nouns (including verbal nouns), although there is some information for verbs and adjectives.

\section{Parse Selection}

Combining the broad-coverage JACY grammar and the Hinoki corpus, we build a parse selection model on top of the symbolic grammar. Given a set of can- 


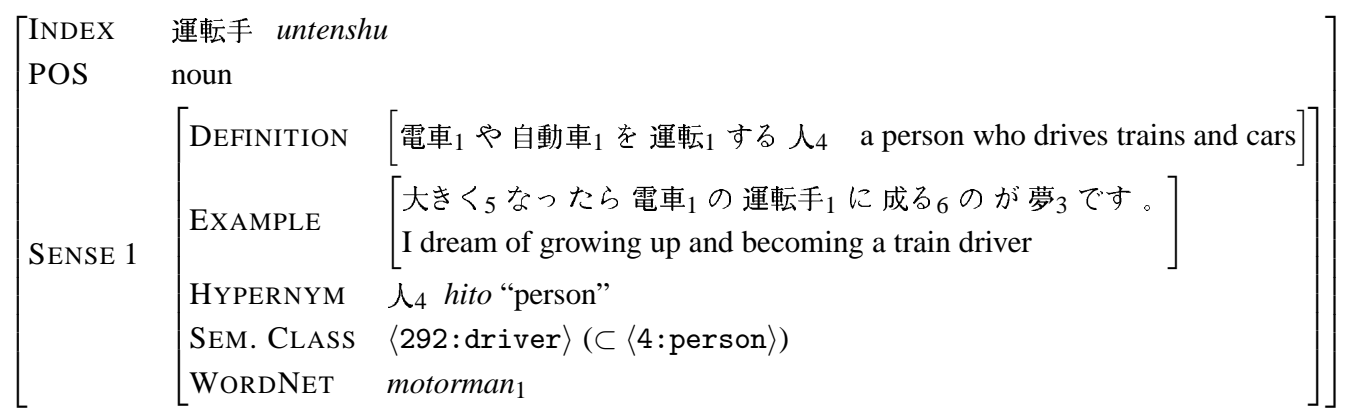

Figure 1: Dictionary Entry for 運転手 ${ }_{1}$ untenshu “chauffeur"

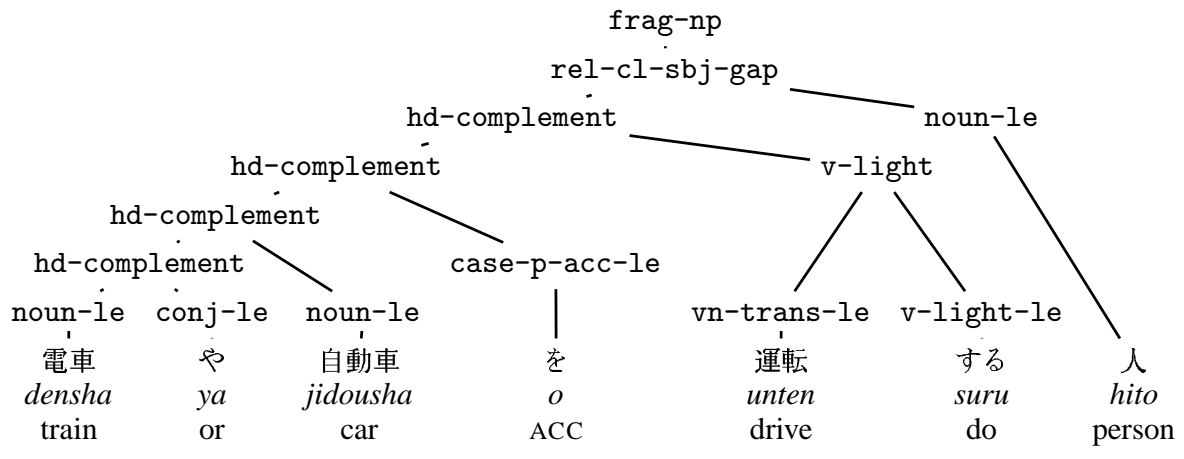

運転手 1 “chauffeur”: “a person who drives a train or car"

Figure 3: Derivation Tree of the Definition of 運転手 ${ }_{1}$ untenshu "chauffeur"

Phrasal nodes are labeled with identifiers of grammar rules, and (pre-terminal) lexical nodes with class names for types of lexical entries.

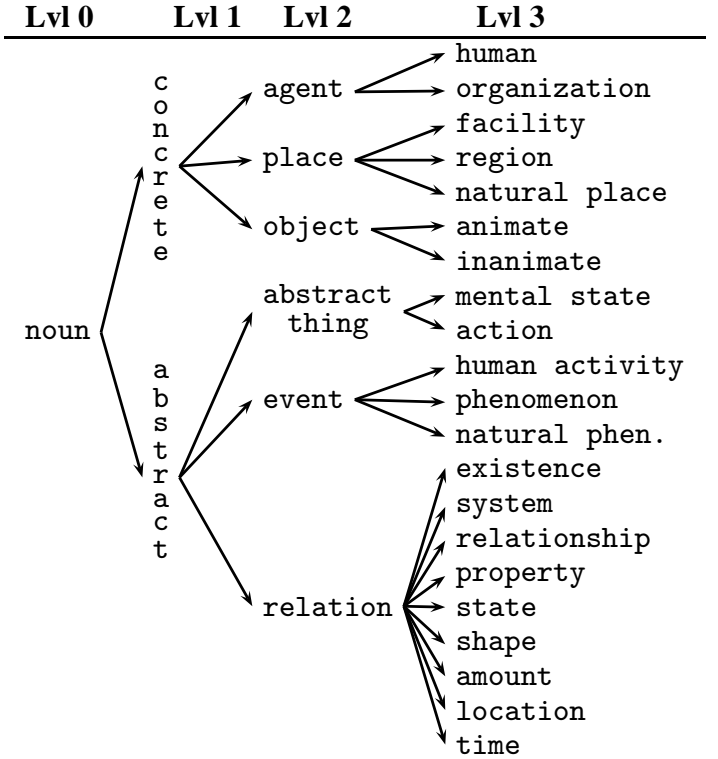

Figure 5: Top 3 levels of the GoiTaikei Ontology

didate analyses (for some Japanese string) according to JACY, the goal is to rank parse trees by their probability: training a stochastic parse selection model on the available treebank, we estimate statistics of various features of candidate analyses from the treebank. The definition and selection of features, thus, is a central parameter in the design of an effective parse selection model.

\subsection{Syntactic Features}

The first model that we trained uses syntactic features defined over HPSG derivation trees as summarized in Table 1. For the closely related purpose of parse selection over the English Redwoods treebank, Toutanova et al. (2005) train a discriminative loglinear model, using features defined over derivation trees with non-terminals representing the construction types and lexical types of the HPSG grammar. The basic feature set of our parse selection model for Japanese is defined in the same way (corresponding to the PCFG-S model of Toutanova et al. (2005)): each feature capturing a sub-tree from the deriva- 


\begin{tabular}{|c|c|}
\hline$\#$ & sample features \\
\hline 1 & $\overline{\langle 0 \text { rel-cl-sbj-gap hd-complement noun-le }\rangle}$ \\
\hline 1 & $\langle 1$ frag-np rel-cl-sbj-gap hd-complement noun-le $\rangle$ \\
\hline 1 & $\langle 2 \triangle$ frag-np rel-cl-sbj-gap hd-complement noun-le $\rangle$ \\
\hline 2 & $\langle 0$ rel-cl-sbj-gap hd-complement $\rangle$ \\
\hline 2 & $\langle 0$ rel-cl-sbj-gap noun-le $\rangle$ \\
\hline 2 & $\langle 1$ frag-np rel-cl-sbj-gap hd-complement $\rangle$ \\
\hline 2 & $\langle 1$ frag-np rel-cl-sbj-gap noun-le $\rangle$ \\
\hline 3 & $\langle 1$ conj-le ya $\rangle$ \\
\hline 3 & $\langle 2$ noun-le conj-le ya $\rangle$ \\
\hline 3 & $\langle 3 \triangleleft$ noun-le conj-le ya $\rangle$ \\
\hline 4 & $\langle 1$ conj-le $\rangle$ \\
\hline 4 & $\langle 2$ noun-le conj-le $\rangle$ \\
\hline 4 & $\langle 3 \triangleleft$ noun-le conj-le $\rangle$ \\
\hline
\end{tabular}

Table 1: Example structural features extracted from the derivation tree in Figure 3. The first column numbers the feature template corresponding to each example; in the examples, the first integer value is a parameter to feature templates, i.e. the depth of grandparenting (types \#1 and\#2) or $n$-gram size (types \#3 and \#4). The special symbols $\triangle$ and $\triangleleft$ denote the root of the tree and left periphery of the yield, respectively.

tion limited to depth one. Table 1 shows example features extracted from our running example (Figure 3 above) in our MaxEnt models, where the feature template \#1 corresponds to local derivation subtrees. We will refer to the parse selection model using only local structural features as SYN-1.

\subsubsection{Dominance Features}

To reduce the effects of data sparseness, feature type \#2 in Table 1 provides a back-off to derivation sub-trees, where the sequence of daughters is reduced to just the head daughter. Conversely, to facilitate sampling of larger contexts than just subtrees of depth one, feature template \#1 allows optional grandparenting, including the upwards chain of dominating nodes in some features. In our experiments, we found that grandparenting of up to three dominating nodes gave the best balance of enlarged context vs. data sparseness. Enriching our basic model SYN-1 with these features we will henceforth call SYN-GP.

\subsubsection{N-Gram Features}

In addition to these dominance-oriented features taken from the derivation trees of each parse tree, our models also include more surface-oriented features, viz. $n$-grams of lexical types with or without lexicalization. Feature type \#3 in Table 1 defines $n$-grams of variable size, where (in a loose analogy to part-of-speech tagging) sequences of lexical types capture syntactic category assignments. Feature templates \#3 and \#4 only differ with regard to lexicalization, as the former includes the surface token associated with the rightmost element of each $n$-gram (loosely corresponding to the emission probabilities in an HMM tagger). We used a maximum $n$-gram size of two in the experiments reported here, again due to its empirically determined best overall performance.

\subsection{Semantic Features}

In order to define semantic parse selection features, we use a reduction of the full semantic representation (MRS) into 'variable-free' elementary dependencies. The conversion centrally rests on a notion of one distinguished variable in each semantic relation. For most types of relations, the distinguished variable corresponds to the main index (ARG0 in the examples above), e.g. an event variable for verbal relations and a referential index for nominals. Assuming further that, by and large, there is a unique relation for each semantic variable for which it serves as the main index (thus assuming, for example, that adjectives and adverbs have event variables of their own, which can be motivated in predicative usages at least), an MRS can be broken down into a set of basic dependency tuples of the form shown in Figure 4 (Oepen and Lønning, 2006).

All predicates are indexed to the position of the word or words that introduced them in the input sentence (<start:end>). This allows us to link them to the sense annotations in the corpus.

\subsubsection{Basic Semantic Dependencies}

The basic semantic model, SEM-Dep, consists of features based on a predicate and its arguments taken from the elementary dependencies. For example, consider the dependencies for densha ya jidoushawo unten suru hito "a person who drives a train or car" given in Figure 4. The predicate unten "drive" has two arguments: ARG1 hito "person" and ARG2 jidousha "car".

From these, we produce several features (See Table 2). One has all arguments and their labels (\#20). We also produce various back offs: \#21 introduces 


\begin{tabular}{|c|l|}
\hline$\#$ & \multicolumn{1}{|c|}{ sample features } \\
\hline \hline 20 & $\langle 0$ _unten_s ARG1 _hito_n_1 ARG2 _ya_p_conj $\rangle$ \\
20 & $\langle 0$ _ya_p_conj LIDX _densha_n_1 RIDX _jidousha_n_1 $\rangle$ \\
21 & $\langle 1$ _unten_s ARG1 _hito_n_1 $\rangle$ \\
21 & $\langle 1$ _unten_s ARG2 _jidousha_n_1 $\rangle$ \\
21 & $\langle 1$-ya_p_conj LIDX_densha_n_1 $\rangle$ \\
21 & $\langle 1$ _ya_p_conj RIDX _jidousha_n_1 $\rangle$ \\
22 & $\langle 2$ _unten_s _hito_n_1 _jidousha_n_1 $\rangle$ \\
23 & $\langle 3$ _unten_s _hito_n_1 $\rangle$ \\
23 & $\langle 3$ _unten_s _jidousha_n_1 $\rangle$ \\
$\ldots$ & \\
\hline
\end{tabular}

Table 2: Example semantic features (SEM-Dep) extracted from the dependency tree in Figure 4.

only one argument at a time, \#22 provides unlabeled relations, \#23 provides one unlabeled relation at a time and so on.

Each combination of a predicate and its related argument(s) becomes a feature. These resemble the basic semantic features used by Toutanova et al. (2005). We further simplify these by collapsing some non-informative predicates, e.g. the unknown predicate used in fragments.

\subsubsection{Word Sense and Semantic Class Dependencies}

We created two sets of features based only on the word senses. For SEM-WS we used the sense annotation to replace each underspecified MRS predicate by a predicate indicating the word sense. This used the gold standard sense tags. For SEM-Class, we used the sense annotation to replace each predicate by its Goi-Taikei semantic class.

In addition, to capture more useful relationships, conjunctions were followed down into the left and right daughters, and added as separate features. The semantic classes for 電車 ${ }_{1}$ densha "train" and 自 動 車 ${ }_{1 j}$ idousha "car" are both $\langle 988$ : land vehicle $\rangle$, while 運転1 unten "drive" is $\langle 2003$ :motion〉 and $\widehat{人}_{4}$ hito "person"is $\langle 4$ : human $\rangle$. The sample features of SEM-Class are shown in Table 3.

These features provide more specific information, in the case of the word sense, and semantic smoothing in the case of the semantic classes, as words are binned into only 2,700 classes.

\subsubsection{Superordinate Semantic Classes}

We further smooth these features by replacing the semantic classes with their hypernyms at a given level (SEM-L). We investigated levels 2 to 5. Pred-

\begin{tabular}{|c|c|}
\hline$\#$ & \multicolumn{1}{|c|}{ sample features } \\
\hline \hline 40 & $\langle 0$-unten_s ARG1 C4 ARG2 C988 $\rangle$ \\
40 & $\langle 1$ C2003 ARG1 C4 ARG2 C988 \\
40 & $\langle 1$ C2003 ARG1 C4 ARG2 C988 $\rangle$ \\
40 & $\langle 0$ _ya_p_conj LIDX C988 RIDX C988 $\rangle$ \\
41 & $\langle 2$-unten_s ARG1 C4 $\rangle$ \\
41 & $\langle 2$-unten_s ARG2 C988 $\rangle$ \\
$\ldots$ & \\
\hline
\end{tabular}

Table 3: Example semantic class features (SEMClass).

icates are binned into only 9 classes at level 2, 30 classes at level 3, 136 classes at level 4, and 392 classes at level 5 .

For example, at level 3, the hypernym class for $\langle 988:$ land vehicle $\rangle$ is $\langle 706:$ inanimate $\rangle$,

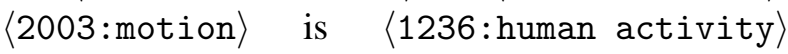
and $\langle 4:$ human $\rangle$ is unchanged. So we used $\langle 706$ : inanimate $\rangle$ and $\langle 1236$ : human activity to make features in the same way as Table 3 .

An advantage of these underspecified semantic classes is that they are more robust to errors in word sense disambiguation - fine grained sense distinctions can be ignored.

\subsubsection{Valency Dictionary Compatability}

The last kind of semantic information we use is valency information, taken from the Japanese side of the Goi-Taikei Japanese-English valency dictionary as extended by Fujita and Bond (2004). This valency dictionary has detailed information about the argument properties of verbs and adjectives, including subcategorization and selectional restrictions. A simplified entry of the Japanese side for 運転す る unten-suru "drive" is shown in Figure 6.

Each entry has a predicate and several case-slots. Each case-slot has information such as grammatical function, case-marker, case-role $(\mathrm{N} 1, \mathrm{~N} 2, \ldots)$ and semantic restrictions. The semantic restrictions are defined by the Goi-Taikei's semantic classes.

On the Japanese side of Goi-Taikei's valency dictionary, there are 10,146 types of verbs giving 18,512 entries and 1,723 types of adjectives giving 2,618 entries.

The valency based features were constructed by first finding the most appropriate pattern, and then recording how well it matched.

To find the most appropriate pattern, we extracted candidate dictionary entries whose lemma is the 


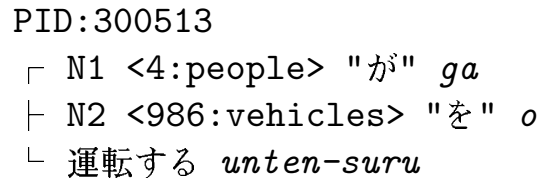

Figure 6: 運転するunten-suru “N1 drive N2". PID is the verb's Pattern ID

\begin{tabular}{|c|c|}
\hline$\#$ & sample features \\
\hline 31 & $\langle 0$ High $\rangle$ \\
\hline 31 & $\langle 1300513$ High $\rangle$ \\
\hline 31 & $\left\langle\begin{array}{ll}2 & 2\rangle\end{array}\right.$ \\
\hline 31 & $\langle 3$ R:High $\rangle$ \\
\hline 31 & $\langle 4300513$ R:High $\rangle$ \\
\hline 32 & $\langle 1$ _unten_s High $\rangle$ \\
\hline 32 & $\langle 4$ _unten_s R:High $\rangle$ \\
\hline 33 & $\langle 5 \mathrm{~N} 1 \mathrm{C}$ High $\rangle$ \\
\hline 33 & $\langle 7 \mathrm{C}\rangle$ \\
\hline
\end{tabular}

Table 4: Example semantic features (SP)

same as the predicate in the sentence: for example we look up all entries for 運転する untensuru "drive". Then, for each candidate pattern, we mapped its arguments to the target predicate's arguments via case-markers. If the target predicate has no suitable argument, we mapped to comitative phrase. Finally, for each candidate patterns, we calculate a matching score ${ }^{1}$ and select the pattern which has the best score.

Once we have the most appropriate pattern, we then construct features that record how good the match is (Table 4). These include: the total score, with or without the verb's Pattern ID (High/Med/Low/Zero: \#31 0,1), the number of filled arguments (\#31 2), the fraction of filled arguments vs all arguments (High/Med/Low/Zero: \#31 3,4), the score for each argument of the pattern (\#32 5) and the types of matches (\#32 5,7).

These scores allow us to use information about word usage in an exisiting dictionary.

\section{Evaluation and Results}

We trained and tested on a subset of the dictionary definition and example sentences in the Hinoki corpus. This consists of those sentences with ambiguous parses which have been annotated so that the

\footnotetext{
${ }^{1}$ The scoring method follows Bond and Shirai (1997), and depends on the goodness of the matches of the arguments.
}

number of parses has been reduced (Table 5). That is, we excluded unambiguous sentences (with a single parse), and those where the annotators judged that no parse gave the correct semantics. This does not necessarily mean that there is a single correct parse, we allow the annotator to claim that two or more parses are equally appropriate.

\begin{tabular}{llrrr} 
Corpus & & \# Sents & $\begin{array}{r}\text { Length } \\
\text { (Ave) }\end{array}$ & $\begin{array}{r}\text { Parses/Sent } \\
\text { (Ave) }\end{array}$ \\
\hline Definitions & Train & 30,345 & 9.3 & 190.1 \\
& Test & 2,790 & 10.1 & 177.0 \\
Examples & Train & 27,081 & 10.9 & 74.1 \\
& Test & 2,587 & 10.4 & 47.3
\end{tabular}

Table 5: Data of Sets for Evaluation

Dictionary definition sentences are a different genre to other commonly used test sets (e.g newspaper text in the Penn Treebank or travel dialogues in Redwoods). However, they are valid examples of naturally occurring texts and a native speaker can read and understand them without special training. The main differences with newspaper text is that the definition sentences are shorter, contain more fragments (especially NPs as single utterances) and fewer quoting and proper names. The main differences with travel dialogues is the lack of questions.

\subsection{A Maximum Entropy Ranker}

Log-linear models provide a very flexible framework that has been widely used for a range of tasks in NLP, including parse selection and reranking for machine translation. We use a maximum entropy / minimum divergence (MEMD) modeler to train the parse selection model. Specifically, we use the open-source Toolkit for Advanced Discriminative Modeling (TADM: ${ }^{2}$ Malouf, 2002) for training, using its limited-memory variable metric as the optimization method and determining best-performing convergence thresholds and prior sizes experimentally. A comparison of this learner with the use of support vector machines over similar data found that the SVMs gave comparable results but were far slower (Baldridge and Osborne, 2007). Because we are investigating the effects of various different features, we chose the faster learner.

\footnotetext{
${ }^{2}$ http://tadm.sourceforge.net
} 


\begin{tabular}{lrrrr} 
Method & \multicolumn{2}{c}{ Definitions } & \multicolumn{2}{c}{ Examples } \\
& $\begin{array}{r}\text { Accuracy } \\
(\%)\end{array}$ & $\begin{array}{r}\text { Features } \\
(\times 1000)\end{array}$ & $\begin{array}{r}\text { Accuracy } \\
(\%)\end{array}$ & $\begin{array}{r}\text { Features } \\
(\times 1000)\end{array}$ \\
\hline SYN-1 & 52.8 & 7 & 67.6 & 8 \\
SYN-GP & 62.7 & 266 & 76.0 & 196 \\
SYN-ALL & $\mathbf{6 3 . 8}$ & 316 & $\mathbf{7 6 . 2}$ & 245 \\
\hline SYN baseline & 16.4 & random & 22.3 & random \\
\hline SEM-Dep & 57.3 & 1,189 & 58.7 & 675 \\
+SEM-WS & 56.2 & 1,904 & 59.0 & 1,486 \\
+SEM-Class & 57.5 & 2,018 & 59.7 & 1,669 \\
+SEM-L2 & 60.3 & 808 & 62.9 & 823 \\
+SEM-L3 & 59.8 & 876 & 62.8 & 879 \\
+SEM-L4 & 59.9 & 1,000 & 62.3 & 973 \\
+SEM-L5 & 60.4 & 1,240 & 61.3 & 1,202 \\
+SP & 59.1 & 1,218 & 68.2 & 819 \\
+SEM-ALL & $\mathbf{6 2 . 7}$ & 3,384 & $\mathbf{6 9 . 1}$ & 2,693 \\
\hline SYN-SEM & $\mathbf{6 9 . 5}$ & 2,476 & $\mathbf{7 9 . 2}$ & 2,126 \\
\hline \hline SEM baseline & 20.3 & random & 22.8 & random
\end{tabular}

Table 6: Parse Selection Results

\subsection{Results}

The results for most of the models discussed in the previous section are shown in Table 6 . The accuracy is exact match for the entire sentence: a model gets a point only if its top ranked analysis is the same as an analysis selected as correct in Hinoki. This is a stricter metric than component based measures (e.g., labelled precision) which award partial credit for incorrect parses. For the syntactic models, the baseline (random choice) is $16.4 \%$ for the definitions and $22.3 \%$ for the examples. Definition sentences are harder to parse than the example sentences. This is mainly because they have fewer relative clauses and coordinate NPs, both large sources of ambiguity. For the semantic and combined models, multiple sentences can have different parses but the same semantics. In this case all sentences with the correct semantics are scored as good. This raises the baselines to 20.3 and $22.8 \%$ respectively.

Even the simplest models (SYN-1 and SEM-Dep) give a large improvement over the baseline. Adding grandparenting to the syntactic model has a large improvement (SYN-GP), but adding lexical n-grams gave only a slight improvement over this (SYN-ALL).

The effect of smoothing by superordinate semantic classes (SEM-Class), shows a modest improvement. The syntactic model already contains a backoff to lexical-types, we hypothesize that the semantic classes behave in the same way. Surprisingly, as we add more data, the very top level of the semantic class hierarchy performs almost as well as the

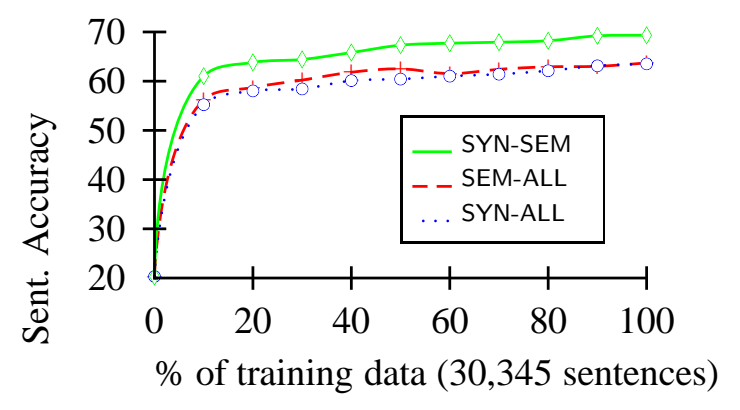

Figure 7: Learning Curves (Definitions)

more detailed levels. The features using the valency dictionary (SP) also provide a considerable improvement over the basic dependencies.

Combining all the semantic features (SEM-ALL) provides a clear improvement, suggesting that the information is heterogeneous. Finally, combing the syntactic and semantic features gives the best results by far (SYN-SEM: SYN-ALL + SEM-Dep + SEM-Class + SEM-L2 + SP). The definitions sentences are harder syntactically, and thus get more of a boost from the semantics. The semantics still improve performance for the example sentences.

The semantic class based sense features used here are based on manual annotation, and thus show an upper bound on the effects of these features. This is not an absolute upper bound on the use of sense information - it may be possible to improve further through feature engineering. The learning curves (Fig 7) have not yet flattened out. We can still improve by increasing the size of the training data.

\section{Discussion}

Bikel (2000) combined sense information and parse information using a subset of SemCor (with WordNet senses and Penn-II treebanks) to produce a combined model. This model did not use semantic dependency relations, but only syntactic dependencies augmented with heads, which suggests that the deeper structural semantics provided by the HPSG parser is important. Xiong et al. (2005) achieved only a very minor improvement over a plain syntactic model, using features based on both the correlation between predicates and their arguments, and between predicates and the hypernyms of their arguments (using HowNet). However, they do not investigate generalizing to different levels than a word's immediate hypernym. 
Pioneering work by Toutanova et al. (2005) and Baldridge and Osborne (2007) on parse selection for an English HPSG treebank used simpler semantic features without sense information, and got a far less dramatic improvement when they combined syntactic and semantic information.

The use of hand-crafted lexical resources such as the Goi-Taikei ontology is sometimes criticized on the grounds that such resources are hard to produce and scarce. While it is true that valency lexicons and sense hierarchies are hard to produce, they are of such value that they have already been created for all of the languages we know of which have large treebanks. In fact, there are more languages with WordNets than large treebanks.

In future work we intend to confirm that we can get improved results with raw sense disambiguation results not just the gold standard annotations and test the results on other sections of the Hinoki corpus.

\section{Conclusions}

We have shown that sense-based semantic features combined with ontological information are effective for parse selection. Training and testing on the definition subset of the Hinoki corpus, a combined model gave a 5.6\% improvement in parse selection accuracy over a model using only syntactic features $(63.8 \% \rightarrow 69.4 \%)$. Similar results $(76.2 \%$ $\rightarrow 79.2 \%$ ) were found with example sentences.

\section{References}

Jason Baldridge and Miles Osborne. 2007. Active learning and logarithmic opinion pools for HPSG parse selection. Natural Language Engineering, 13(1):1-32.

Daniel M. Bikel. 2000. A statistical model for parsing and word-sense disambiguation. In Proceedings of the Joint SIGDAT Conference on Empirical Methods in Natural Language Processing and Very Large Corpora, pages 155-163. Hong Kong.

Francis Bond, Sanae Fujita, and Takaaki Tanaka. 2007. The Hinoki syntactic and semantic treebank of Japanese. Language Resources and Evaluation. (Special issue on Asian language technology).

Francis Bond and Satoshi Shirai. 1997. Practical and efficient organization of a large valency dictionary. In Workshop on Multilingual Information Processing - Natural Language Processing Pacific Rim Symposium '97: NLPRS-97. Phuket.

Ann Copestake, Dan Flickinger, Carl Pollard, and Ivan A. Sag. 2005. Minimal Recursion Semantics. An introduction. Research on Language and Computation, 3(4):281-332.

Christine Fellbaum, editor. 1998. WordNet: An Electronic Lexical Database. MIT Press.
Sanae Fujita and Francis Bond. 2004. A method of creating new bilingual valency entries using alternations. In Gilles Sérasset, editor, COLING 2004 Multilingual Linguistic Resources, pages 41-48. Geneva.

Eduard Hovy, Mitchell Marcus, Martha Palmer, Lance Ramshaw, and Ralph Weischedel. 2006. Ontonotes: The 90\% solution. In Proceedings of the Human Language Technology Conference of the NAACL, Companion Volume: Short Papers, pages 57-60. Association for Computational Linguistics, New York City, USA. URL http://www . aclweb.org/anthology/N/N06/N06-2015.

Satoru Ikehara, Masahiro Miyazaki, Satoshi Shirai, Akio Yokoo, Hiromi Nakaiwa, Kentaro Ogura, Yoshifumi Ooyama, and Yoshihiko Hayashi. 1997. Goi-Taikei A Japanese Lexicon. Iwanami Shoten, Tokyo. 5 volumes/CDROM.

Kaname Kasahara, Hiroshi Sato, Francis Bond, Takaaki Tanaka, Sanae Fujita, Tomoko Kanasugi, and Shigeaki Amano. 2004. Construction of a Japanese semantic lexicon: Lexeed. In IPSG SIG: 2004-NLC-159, pages 75-82. Tokyo. (in Japanese).

Dan Klein and Christopher D. Manning. 2003. Accurate unlexicalized parsing. In Erhard Hinrichs and Dan Roth, editors, Proceedings of the 41st Annual Meeting of the Association for Computational Linguistics, pages 423-430. URL http://www. aclweb.org/anthology/P03-1054.pdf.

Robert Malouf. 2002. A comparison of algorithms for maximum entropy parameter estimation. In CONLL-2002, pages 49-55. Taipei, Taiwan.

Robert Malouf and Gertjan van Noord. 2004. Wide coverage parsing with stochastic attribute value grammars. In IJCNLP-04 Workshop: Beyond shallow analyses - Formalisms and statistical modeling for deep analyses. JST CREST. URL http://www-tsujii.is.s.u-tokyo.ac. jp/bsa/papers/malouf .pdf.

Stephan Oepen, Dan Flickinger, Kristina Toutanova, and Christoper D. Manning. 2004. LinGO redwoods: A rich and dynamic treebank for HPSG. Research on Language and Computation, 2(4):575-596.

Stephan Oepen and Jan Tore Lønning. 2006. Discriminantbased MRS banking. In Proceedings of the 5th International Conference on Language Resources and Evaluation (LREC 2006). Genoa, Italy.

Carl Pollard and Ivan A. Sag. 1994. Head Driven Phrase Structure Grammar. University of Chicago Press, Chicago.

Melanie Siegel and Emily M. Bender. 2002. Efficient deep processing of Japanese. In Proceedings of the 3rd Workshop on Asian Language Resources and International Standardization at the 19th International Conference on Computational Linguistics, pages 1-8. Taipei.

Kristina Toutanova, Christopher D. Manning, Dan Flickinger, and Stephan Oepen. 2005. Stochastic HPSG parse disambiguation using the redwoods corpus. Research on Language and Computation, 3(1):83-105.

Deyi Xiong, Qun Liu Shuanglong Li and, Shouxun Lin, and Yueliang Qian. 2005. Parsing the Penn Chinese treebank with semantic knowledge. In Robert Dale, Jian Su Kam-Fai Wong and, and Oi Yee Kwong, editors, Natural Language Processing - IJCNLP 005: Second International Joint Conference Proceedings, pages 70-81. Springer-Verlag. 